\title{
Forward vs. Bayesian Inference Parameter Calibration: Two Approaches for Non-deterministic Parameter Calibration of a Beam-Column Model
}

\author{
Maximilian Schaeffner ${ }^{1(\bowtie)}$, Christopher M. Gehb ${ }^{1}$, Robert Feldmann ${ }^{1}$, \\ and Tobias Melz ${ }^{1,2}$ \\ 1 Mechanical Engineering Department, System Reliability, \\ Adaptive Structures and Machine Acoustics SAM, \\ Technical University of Darmstadt, Darmstadt, Germany \\ maximilian.schaeffner@sam.tu-darmstadt.de \\ ${ }^{2}$ Fraunhofer Institute for Structural Durability and System Reliability LBF, \\ Darmstadt, Germany
}

\begin{abstract}
Mathematical models are commonly used to predict the dynamic behavior of mechanical structures or to synthesize controllers for active systems. Calibrating the model parameters to experimental data is crucial to achieve reliable and adequate model predictions. However, the experimental dynamic behavior is uncertain due to variations in component properties, assembly and mounting. Therefore, uncertainty in the model parameters can be considered in a non-deterministic calibration. In this paper, we compare two approaches for a non-deterministic parameter calibration, which both consider uncertainty in the parameters of a beam-column model. The goal is to improve the model prediction of the axial load-dependent lateral dynamic behavior. The investigation is based on a beam-column system subjected to compressive axial loads used for active buckling control. A representative sample of 30 nominally identical beam-column systems characterizes the variations in the experimental lateral axial load-dependent dynamic behavior. First, in a forward parameter calibration approach, the parameters of the beamcolumn model are calibrated separately for all 30 investigated beamcolumn systems using a least squares optimization. The uncertainty in the parameters is obtained by assuming normal distributions of the separately calibrated parameters. Second, in a Bayesian inference parameter calibration approach, the parameters are calibrated using the complete sample of experimental data. Posterior distributions of the parameters characterize the uncertain dynamic behavior of the beam-column model. For both non-deterministic parameter calibration approaches, the predicted uncertainty ranges of the axial load-dependent lateral dynamic behavior are compared to the uncertain experimental behavior and the most accurate results are identified.
\end{abstract}

Keywords: Bayesian inference - Uncertainty - Parameter calibration • Frequency response $\cdot$ Active buckling control 


\section{Introduction}

In engineering science, mathematical models are of utmost importance to predict the dynamic behavior of structures or to improve the structural design. The necessity for accurate mathematical models arises from the ever-increasing virtualization of the product development process and the need to assess dynamic performance under all possible environmental and operating conditions for stability evaluation, designing robust structures or for appropriate controller design [1-3]. During the modeling process, assumptions and simplifications have to be made that determine the form of a model and its parameters. Thereby, model uncertainty and parameter uncertainty are almost unavoidable and omnipresent [4]. Consequently, the numerical predictions of the mathematical model are also uncertain. It follows that the quantification and reduction of model and parameter uncertainty is necessary for reliable and adequate numerical predictions. In this paper and as a gambit for reliable and adequate numerical predictions, we focus on reducing parameter uncertainty while shelving the consideration of model uncertainty. Reducing the parameter uncertainty is achieved i.e. via model parameter calibration [5-7]. Thus, the mathematical model and its numerical predictions are adjusted to the experimentally observed dynamic behavior.

Parameter calibration is commonly achieved by solving an optimization problem to find deterministic values for each model parameter to be calibrated that best fit the chosen calibration criteria. For example in [8], parameters of a friction model were identified for a direct-drive rotary torque motor using the Novel Evolutionary Algorithm optimization. Experimental data from a test rig was used within the optimization process and two objective functions were minimized for different parameter sets. As a result, the calibrated parameters are stated as deterministic values. Similar approaches but using for example genetic and particle swarm optimization algorithms for parameter calibration can be found in [6] and [9] for models of mechanical servo systems. In these studies, the remaining parameter uncertainty after calibration is not taken into account. Hence, deterministic optimization approaches are searching for the best fitting parameter values and then treating the parameters as known and fixed.

In contrast, non-deterministic calibration approaches aim to achieve statistical consistency between model prediction and experimental data $[10,11]$. The calibrated parameters are stated as distributions representing the remaining parameter uncertainty. In [12], the model parameters of a historic masonry monument FE model were calibrated using a non-deterministic calibration approach. BAYESIAN inference parameter calibration with MARKOV CHAIN MONTE CARLO leads to calibrated parameters resulting in reduced uncertainty in the model prediction. Another example for non-deterministic parameter calibration can be found in [5]. BAYESIAN inference was successfully used to calibrate parameters for several friction models, but inconclusive for the parameters of a LUGREfriction model. Successful use of BAYESIAN inference to calibrate parameters of a LUGRE-friction model was conducted in [13]. Although only 3 out of 7 model 
parameters could be calibrated, the uncertainty of the model prediction was reduced considerably.

In this paper, the parameters of a beam-column system subjected to axial loading and prone to buckling, [14,15], are calibrated using two different approaches for non-deterministic parameter calibration, namely a forward parameter calibration approach [14] and a BAYESIAN inference parameter calibration approach $[10,16]$. The calibrated beam-column model is used to design an active buckling control, which is intended to increase the maximum bearable load of the beam-column $[14,15]$. The accuracy and credibility of the mathematical beam-column model is essential for the successful application of the active buckling control.

The experimental data for the parameter calibration of the beam-column model is obtained by measuring the axial load-dependent lateral dynamic behavior of 30 nominally identical beam-column systems subjected to varying axial loading. In the forward parameter calibration approach, the parameters of the beam-column model are calibrated separately for all 30 investigated beamcolumn systems using a least squares optimization. The uncertainty in the parameters is obtained by assuming normal distributions of the separately calibrated parameters. The non-deterministic BAYESIAN inference parameter calibration approach statistically correlates the beam-column model predictions with the experimental data. Additionally, it enables to quantify and reduce the parameter uncertainty concurrently. The uncertainty in the parameters is obtained by sampling from the posterior distributions via MARKOV CHAIN Monte CARLO (MCMC) sampling. Both approaches increase the beam-column model prediction credibility since the parameters are not assumed as deterministic and, hence, better represent the typically non-deterministic reality.

This paper is organized as follows: The beam-column system and its corresponding mathematical model are introduced in Sect. 2 and Sect. 3. The model calibration approaches are performed and compared in Sect. 4. Additionally, the model predictions with non-calibrated and calibrated parameters are presented. Finally, conclusions and proposed future work are given in Sect. 5 .

\section{System Description}

This section introduces the investigated beam-column system used for active buckling control in $[14,15]$. In the concept for active buckling control, a slender beam-column subjected to a compressive axial load $F_{x}$ is stabilized by two piezoelastic supports at both beam-column ends, as depicted in the schematic sketch in Fig. 1. 


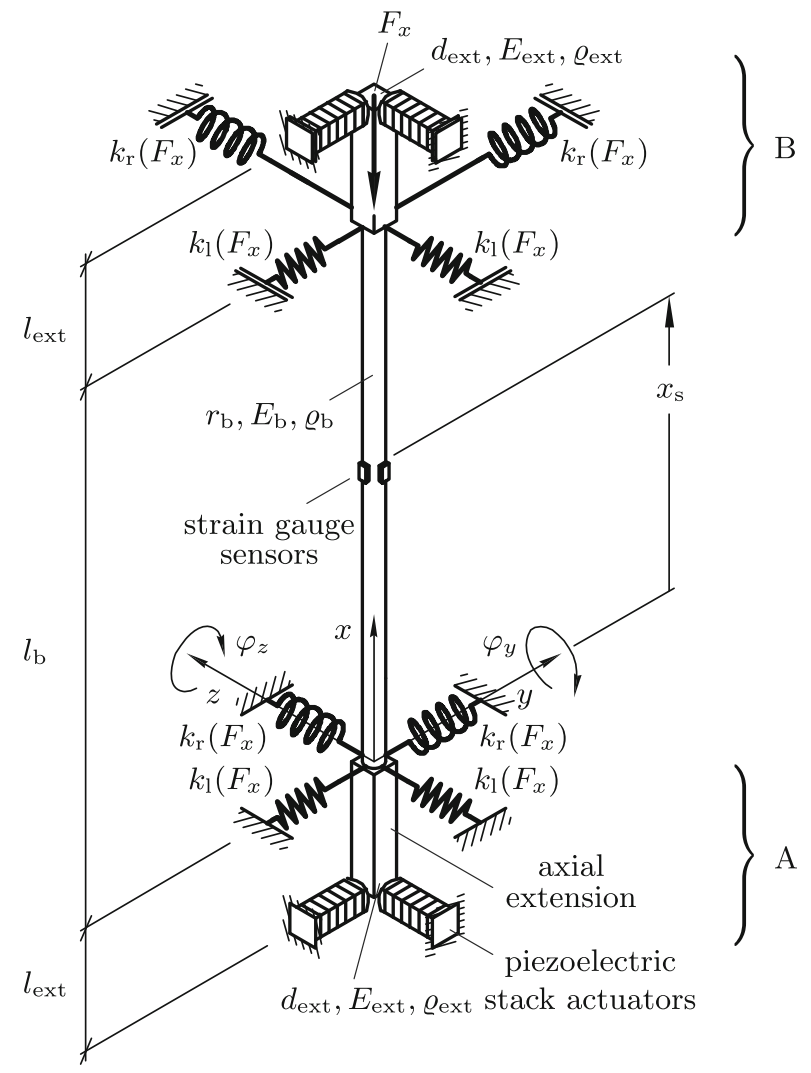

Fig. 1. Schematic sketch of the axially loaded beam-column with elastic supports, piezoelectric stack actuators and strain gauge sensors [14]

The slender beam-column has length $l_{\mathrm{b}}=400 \mathrm{~mm}$, circular solid cross-section with constant radius $r_{\mathrm{b}}=4 \mathrm{~mm}$, Young's modulus $E_{\mathrm{b}}=75.8 \cdot 10^{3} \mathrm{~N} / \mathrm{mm}^{2}$ and density $\varrho_{\mathrm{b}}=2.79 \cdot 10^{-3} \mathrm{~g} / \mathrm{mm}^{3}$. The lower beam-column end is fixed at support A. The upper beam-column end at support B is free to move in longitudinal $x$-direction and is used to apply the axial load $F_{x}$. The piezoelectric stack actuators are integrated in the lateral load path via axial extensions (subscript ext) with length $l_{\text {ext }}=8.1 \mathrm{~mm}$, which are connected to the beam-column ends. The axial extensions have quadratic cross-sections with edge length $d_{\text {ext }}=12 \mathrm{~mm}$ and relatively high bending stiffness with Young's modulus $E_{\text {ext }}=210.0 \cdot 10^{3} \mathrm{~N} / \mathrm{mm}^{2}$ and density $\varrho_{\text {ext }}=7.81 \cdot 10^{-3} \mathrm{~g} / \mathrm{mm}^{3}$. Piezoelectric stack actuators exert active lateral forces in positive and negative $y$ - and $z$-direction to the beam-column's axial extensions by applying the actuator voltages $V_{\mathrm{pz}, y / z}(t)$. The resulting active bending moments may act in arbitrary directions at the lower and upper beamcolumn ends. This is realized by piezo-elastic supports $\mathrm{A}$ at location $x=0$ and $\mathrm{B}$ at $x=l_{\mathrm{b}}$ [17]. Finally, four strain gauge sensors at the sensor position $x_{\mathrm{s}}=l_{\mathrm{b}} / 2$ 
are used to measure the surface strains $\varepsilon_{\mathrm{s}, y / z}(t)$ due to bending to calculate the lateral deflection of the beam-column in $y$ - and $z$-direction.

Figure 2a) shows the experimental beam-column with circular solid crosssection. Its lower and upper ends are connected to the piezo-elastic supports A and B, shown in detail in Fig. 2b), which are fixed to a baseplate and a parallel guidance, respectively.

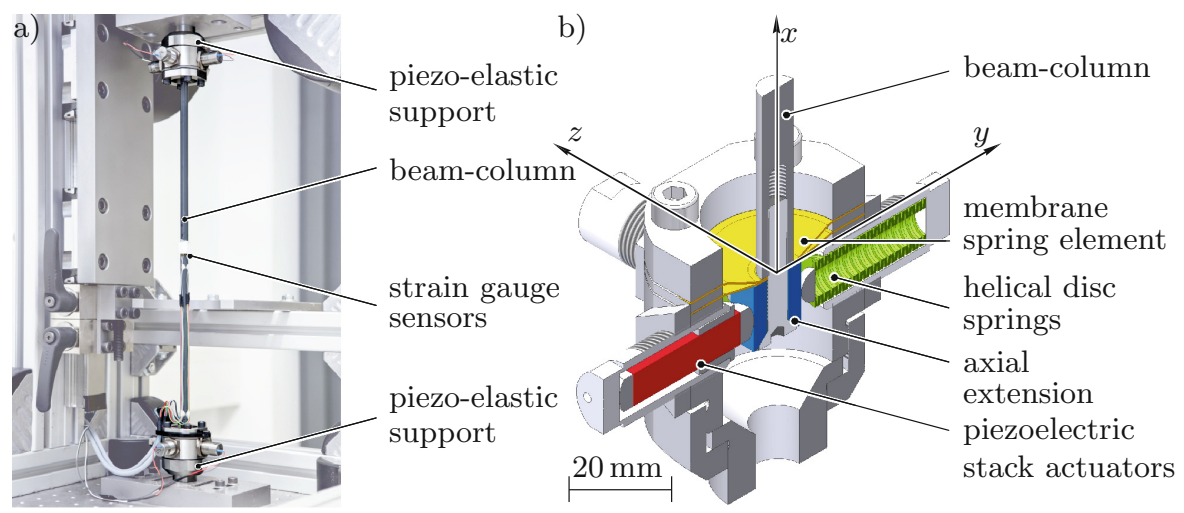

Fig. 2. a) Beam-column system for active buckling control, b) sectional view of piezoelastic support with $x$-, $y$-and $z$-directions [14]

The piezo-elastic supports are designed to provide the elastic boundary conditions of the beam-column and to include the piezoelectric stack actuators to influence the lateral deflections [17]. The elastic boundary conditions are represented by the axial-load-dependent lateral stiffness $k_{1}\left(F_{x}\right)$ and rotational stiffness $k_{\mathrm{r}}\left(F_{x}\right)$ in Fig. 1. Figure $\left.2 \mathrm{~b}\right)$ shows a sectional view of the piezo-elastic support A. Two differently shaped membrane spring elements bear the axial and lateral loads and allow rotations in any plane perpendicular to the beam-column's longitudinal $x$-axis. The two piezoelectric stack actuators exert lateral forces in $y$ - and $z$-direction to the beam-column's axial extensions at a distance $l_{\text {ext }}$ from the beam-column ends, as shown in Fig. 1. The piezoelectric stack actuators are mechanically prestressed by allocated helical disk springs.

The lateral dynamic behavior of the beam-column system is strongly dependent on the axial load $F_{x}(t)$. For the experimental characterization of the axial load-dependent lateral dynamic behavior without active buckling control, the beam-column system is loaded by static axial loads $F_{x}(t)=$ const. and separately excited by broadband white noise via the piezoelectric stack actuator voltages $V_{\mathrm{pz}, y / z}(t)$ in $y$ - and $z$-direction, which results in the measured beam-column surface strains $\varepsilon_{\mathrm{s}, y / z}(t)$. The experimental beam-column transfer functions

$$
G_{y}^{\mathcal{D}}\left(F_{x}, \Omega\right)=\frac{\varepsilon_{\mathrm{s}, y}\left(F_{x}, \Omega\right)}{V_{\mathrm{pz}, y}(\Omega)} \quad \text { and } \quad G_{z}^{\mathcal{D}}\left(F_{x}, \Omega\right)=\frac{\varepsilon_{\mathrm{s}, z}\left(F_{x}, \Omega\right)}{V_{\mathrm{pz}, z}(\Omega)}
$$


in $y$ - and $z$-direction are obtained from the experimental data (superscript $\mathcal{D}$ ) for a harmonic excitation with angular frequency $\Omega$ [18]. The experimental beamcolumn transfer functions $G_{y / z}^{\mathcal{D}}\left(F_{x}, \Omega\right)(1)$ are estimated by the tfestimate algo-

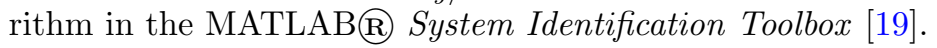

\section{Mathematical Model of the Active Beam-Column}

As in many applications relying on model predictions, the performance of the active buckling control is primarily determined by the quality of the underlying mathematical model used for the model-based controller synthesis, as e.g. in $[14,15]$. In particular, the model of the beam-column system shown in Figs. 1 and 2 has to properly describe the complex boundary conditions created by the piezo-elastic supports and has to include the piezoelectric stack actuators and strain gauge sensors. Furthermore, the axial load-dependency of the beamcolumn that is prone to buckle has to be considered. This section introduces the finite element (FE) model of the beam-column with piezo-elastic supports, actuators and sensors as well as the beam-column transfer function that is later used for parameter calibration in Sect. 4 .

The mathematical FE model of the slender beam-column with piezo-elastic supports used for active buckling control in an experimental test setup is derived in detail in $[14,15]$. The resulting state space beam-column model in the LAPLACE domain according to [18] is given by

$$
\begin{aligned}
s \boldsymbol{x}(s) & =\boldsymbol{A}\left(F_{x}\right) \boldsymbol{x}(s)+\boldsymbol{B} \boldsymbol{u}(s) \\
\boldsymbol{y}(s) & =\boldsymbol{C}_{y} \boldsymbol{x}(s) .
\end{aligned}
$$

The state vector $\boldsymbol{x}(t)$ of the FE beam-column model is composed of the FE displacement vector and its derivative and describes the lateral deflection of the slender beam-column along the $x$-axis.

The axial load-dependent system matrix $\boldsymbol{A}\left(F_{x}\right)$ contains the FE mass matrix, FE damping matrix and the FE stiffness matrix. Damping is modeled by RAYLEIGH proportional damping [20], and the boundary conditions resulting from the piezo-elastic supports and the surrounding structure, see Fig. 1, are included in the FE stiffness matrix, which is linearly dependent on the axial load $F_{x}$ [14]. Potential non-linearities and resulting model uncertainty, especially concerning the membrane spring elements, are purposefully neglected since the linear dependency of the system matrix $\boldsymbol{A}\left(F_{x}\right)$ is required for the concept of the gain-scheduled $H_{\infty}$ controller used for active buckling control.

The external forces of the piezoelectric stack actuators acting on the beamcolumn are modeled by the term $\boldsymbol{B} \boldsymbol{u}(s)$ in (2), where the voltage input matrix $\boldsymbol{B}$ allocates the piezoelectric stack actuator forces to the lateral degrees of freedom of the first and last nodes of the FE model. The beam-column input vector

$$
\boldsymbol{u}(t)=\left[\begin{array}{l}
V_{\mathrm{pz}, y}(t) \\
V_{\mathrm{pz}, z}(t)
\end{array}\right] .
$$


includes the actuator voltages $V_{\mathrm{pz}, y}(t)$ and $V_{\mathrm{pz}, z}(t)$ that are simultaneously applied to both piezoelectric stack actuators in supports $\mathrm{A}$ and $\mathrm{B}$ in $y$ - and $z$-direction. According to Fig. 1, the strain gauge sensors at sensor position $x_{\mathrm{s}}=l_{\mathrm{b}} / 2$ measure the surface strains $\varepsilon_{\mathrm{s}, y}(t)$ and $\varepsilon_{\mathrm{s}, z}(t)$ in the beam-column center due to bending in $y$ - and $z$-direction. Consequently, the surface strains are used as the beam-column model output

$$
\boldsymbol{y}(t)=\left[\begin{array}{l}
\varepsilon_{\mathrm{s}, y}(t) \\
\varepsilon_{\mathrm{s}, z}(t)
\end{array}\right]
$$

calculated from the state vector $\boldsymbol{x}(t)$ and the output matrix $\boldsymbol{C}_{y}$ according to (2), as derived in [14].

Equation (2) is the state space representation of the beam-column model that is used to derive the beam-column transfer function and to analyze the lateral dynamic behavior of the beam-column system. The $[2 \times 2]$ matrix of beam-column model (superscript $\mathcal{M}$ ) transfer functions

$$
\boldsymbol{G}^{\mathcal{M}}\left(F_{x}, s\right)=\left[\begin{array}{cc}
G_{y}^{\mathcal{M}}\left(F_{x}, s\right) & 0 \\
0 & G_{z}^{\mathcal{M}}\left(F_{x}, s\right)
\end{array}\right]=\frac{\boldsymbol{y}\left(F_{x}, s\right)}{\boldsymbol{u}(s)}=\boldsymbol{C}\left(\mathbf{I} s-\boldsymbol{A}\left(F_{x}\right)\right)^{-1} \boldsymbol{B}
$$

describes the transfer behavior from the actuator voltages $\boldsymbol{u}(s)$ (3) to the beamcolumn strains (4) $\boldsymbol{y}(s)[21]$.

\section{Model Parameter Calibration Approaches}

This section presents two non-deterministic calibration approaches for calibrating the parameters of the beam-column model introduced in Sect. 3. Calibrating parameters with experimental data is necessary to adjust the beam-column model to the experimental data, i.e. the measured lateral dynamic behavior, and to achieve a reliable and adequate model prediction.

With both presented approaches, not only one optimally fitted model parameter set is achieved. Instead, parameter uncertainty is taken into account and quantified. The parameters of the beam-column model are calibrated by comparison of the experimental transfer functions (1) with the numerical beam-column model transfer functions (5). With zero initial conditions and the conversion $s=j \Omega$ for the beam-column model (5), [18], the experimental and the beamcolumn model transfer functions are

$$
G^{\mathcal{D}}\left(F_{x}, \Omega\right)=\frac{\varepsilon_{\mathrm{s}}\left(F_{x}, \Omega\right)}{V_{\mathrm{pz}}(\Omega)} \quad \text { and } \quad G^{\mathcal{M}}\left(F_{x}, \Omega\right)=\frac{y\left(F_{x}, \Omega, \boldsymbol{\theta}\right)}{u(\Omega, \boldsymbol{\theta})},
$$

which are obtained for the six measured axial loads $F_{x, 1}=337 \mathrm{~N}, F_{x, 2}=500 \mathrm{~N}$, $F_{x, 3}=1000 \mathrm{~N}, F_{x, 4}=1500 \mathrm{~N}, F_{x, 5}=2000 \mathrm{~N}$ and $F_{x, 6}=2500 \mathrm{~N}$. The lowest axial load that is realizable due to the dead weight of the test setup in Fig. 2a) is $F_{x, 1}=337 \mathrm{~N}$. As was shown in $[14,22]$, the lateral dynamic behavior of the beamcolumn system in $y$ - and $z$-direction is very similar, so that the parameters in both directions and both piezo-elastic supports are assumed to be identical and 
are calibrated as one, see Fig. 1 . Hence, no distinction between $y$ - and $z$-direction is made for the calibration approaches.

The parameters that were shown to have a strong influence on the beamcolumn lateral dynamic behavior, [14,23], are calibrated to fit the numerical beam-column model transfer functions $G^{\mathcal{M}}\left(F_{x}, \Omega\right)$ with the experimental beamcolumn transfer functions $G^{\mathcal{D}}\left(F_{x}, \Omega\right)$, see (6). In addition to [23], a variancebased sensitivity analysis is applied to further reduce the parameter space in order to avoid identifiability issues with parameters [12,13]. The calibrated parameters are

$$
\boldsymbol{\theta}=\left[k_{\mathrm{r}, \mathrm{e}}, k_{\mathrm{r}, \mathrm{g}}, \beta, \zeta_{1}, m_{\mathrm{s}}, k_{\mathrm{s}}\right]
$$

with the elastic and geometric rotational support stiffness $k_{\mathrm{r}, \mathrm{e}}$ and $k_{\mathrm{r}, \mathrm{g}}$ that describe the axial-load dependent rotational support stiffness shown in Fig. 1 via $k_{\mathrm{r}}\left(F_{x}\right)=k_{\mathrm{r}, \mathrm{e}}+F_{x} \cdot k_{\mathrm{g}, \mathrm{r}}$, the piezoelectric force constant $\beta$, the modal damping ratio of the first mode of vibration $\zeta_{1}$, and the sensor mass and stiffness $m_{\mathrm{s}}$ and $k_{\mathrm{s}}$. The parameters of the beam-column model in (2) that are assumed to be fixed for all investigated beam-column systems are given in [14].

\subsection{Forward Parameter Calibration}

Parameter calibration is commonly achieved by solving an optimization problem to find deterministic values for each parameter to be calibrated that best fit the chosen calibration criteria. The experimental data for calibration mostly stems from one investigated system. In contrast, solving multiple optimization problems for varying but nominally identical beam-column systems complements statistical information and provides the opportunity to quantify the parameter uncertainty, as was shown in [14]. In this investigation, the forward parameter calibration is performed for 30 nominally identical beam-column systems that originate from the combination of five identically instrumented beam-columns, three combinations of lower and upper piezo-elastic supports and two different sets of piezoelectric stack actuators. Thus, the applied component variation combines the effects of uncertainty in manufacturing, assembly and mounting of the beam-column systems. For each of the 30 beam-column systems, the parameters $\boldsymbol{\theta}(7)$ in $y$-and $z$-direction are varied to solve the least squares curve fitting problem

$$
\min _{\boldsymbol{\theta}}\left(\sum_{i=1}^{6}\left\|G^{\mathcal{D}}\left(F_{x, i}, \Omega\right)-G^{\mathcal{M}}\left(F_{x, i}, \Omega, \boldsymbol{\theta}\right)\right\|_{2}^{2}\right)
$$

with the experimental and numerical beam-column transfer functions $G^{\mathcal{D}}\left(F_{x, i}, \Omega\right)$ and $G^{\mathcal{M}}\left(F_{x, i}, \Omega, \boldsymbol{\theta}\right)$ from $(6)$ for the six measured axial loads $F_{x, i}$. The model calibration is performed for all six measured axial loads $F_{x}$ at once so that the axial load-dependency is well captured by the model. The least squares curve fitting problem (8) is solved by the lsqnonlin algorithm in the MATLAB(R) Optimization Toolbox [24] by varying the parameters to be calibrated $\boldsymbol{\theta}$ within a specified range, i.e. the parameters' prior bounds. The resulting 30 separately calibrated parameter sets for the $y$ - and $z$-directions are combined in one sample of $N=60$ calibrated parameter sets shown as histograms in Fig. 3. 

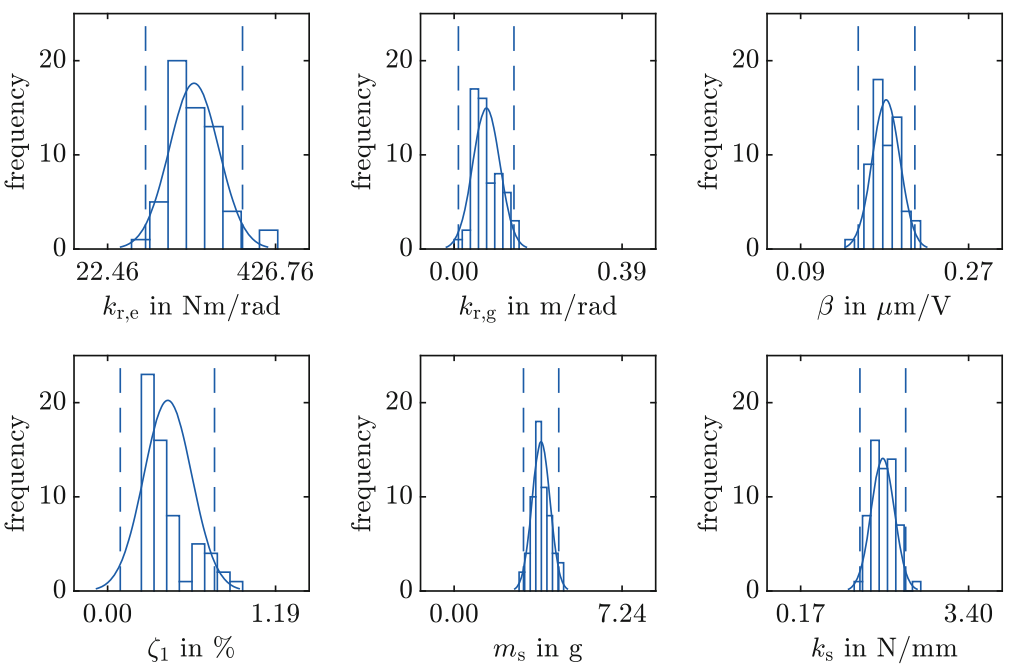

Fig. 3. Histograms of the $N=60$ calibrated parameter sets $\boldsymbol{\theta}$ and normal distribution fits $p_{\mathcal{N}}(\boldsymbol{\theta})$ with $95 \%$ interpercentiles $(--)$

The sample of calibrated parameter sets are approximated by normal distributions (subscript $\mathcal{N}$ ) that are used to describe the parameter uncertainty associated with each parameter of the six calibrated parameters $\boldsymbol{\theta}$. Hence, the forward parameter calibration results in the parameter distributions The fitted normal probability density functions (pdf) $p_{\mathcal{N}}(\boldsymbol{\theta})$ as well as the $95 \%$ interpercentiles given by $\mu_{\boldsymbol{\theta}} \pm 1.96 \sigma_{\boldsymbol{\theta}}$ are also shown in Fig. 3 . The corresponding mean values $\mu_{\boldsymbol{\theta}}$ and standard deviations $\sigma_{\boldsymbol{\theta}}$ are summarized in Table 1.

Table 1. Normal distribution fits for the $N=60$ calibrated parameter sets $\boldsymbol{\theta}$

\begin{tabular}{l|l|l}
\hline Parameter & $\mu_{\theta}$ & $\sigma_{\theta}$ \\
\hline$k_{\mathrm{r}, \mathrm{e}}$ in $\mathrm{Nm} / \mathrm{rad}$ & 230.52 & 59.55 \\
\hline$k_{\mathrm{r}, \mathrm{g}}$ in $\mathrm{m} / \mathrm{rad}$ & 0.08 & 0.03 \\
\hline$\beta$ in $\mu \mathrm{m} / \mathrm{V}$ & 0.18 & 0.01 \\
\hline$\zeta_{1}$ in $\%$ & 0.43 & 0.17 \\
\hline$m_{\mathrm{s}}$ in $\mathrm{g}$ & 3.75 & 0.39 \\
\hline$k_{\mathrm{s}}$ in $\mathrm{N} / \mathrm{mm}$ & 1.75 & 0.22 \\
\hline
\end{tabular}

\subsection{Bayesian Inference Parameter Calibration}

BAYESIAN inference can be used as non-deterministic calibration approach to calibrate uncertain parameters by correlating the model predictions with experimental data, i.e. a number of $n=1, \cdots, N$ measurements, by solving an inverse 
problem $[25,26]$. In this paper, the BAYESIAN inference approach statistically connects the beam-column model and the experimental data (6) to adequately calibrate the varied parameters $\boldsymbol{\theta}(7)$. Current knowledge of the system and its parameters is updated with new information obtained from experimental tests. Thus, the parameter uncertainty is reduced and quantified by inference from the posterior distribution [10,25]. Using BAYES' Theorem [25,27], the posterior parameter distribution given the experimental results can be stated as

$$
P(\boldsymbol{\theta}, \mathcal{M} \mid \mathcal{D})=\frac{L(\mathcal{D} \mid \boldsymbol{\theta}, \mathcal{M}) \times P(\boldsymbol{\theta})}{P(\mathcal{D})} \propto L(\mathcal{D} \mid \boldsymbol{\theta}, \mathcal{M}) \times P(\boldsymbol{\theta}) .
$$

Since the denominator $P(\mathcal{D})$ in $(9)$, the total probability or evidence, is typically not computable with reasonable effort and is only normalizing the result anyway [5], it is more practical to sample from a proportional relationship of the posterior parameter distribution $P(\boldsymbol{\theta}, \mathcal{M} \mid \mathcal{D})$. If no further information regarding the prior distributions $P(\boldsymbol{\theta})$ in (9) is available, uninformative priors between certain upper and lower bounds are assumed $[5,12]$. The likelihood function

$$
L(\mathcal{D} \mid \boldsymbol{\theta}, \mathcal{M})=c^{-1} \prod_{n=1}^{N} \exp \left(E_{\text {stat }, n}(\boldsymbol{\theta})+E_{\max , n}(\boldsymbol{\theta})+E_{\text {ef }, n}(\boldsymbol{\theta})\right)
$$

represents the probability of observing experimental data $\mathcal{D}$ given a set of parameters $\boldsymbol{\theta}$ for the mathematical model $\mathcal{M}[11,25]$. Here, the beam-column transfer functions obtained from the experimental data are defined as $\mathcal{D}:=G^{\mathcal{D}}\left(F_{x}, \Omega\right)$ and the beam-column model transfer functions as $\mathcal{M}:=G^{\mathcal{M}}\left(F_{x}, \Omega, \boldsymbol{\theta}\right)$ according to (6). The scaling factor $c^{-1}$ is constant and crosses out when calculating the acceptance ratio, see Algorithm 1. In this paper, the error function as an essential element of the likelihood function $L(\mathcal{D} \mid \boldsymbol{\theta}, \mathcal{M})$ in $(10)$ consists of the three parts

$$
\begin{aligned}
& E_{\text {stat }, n}(\boldsymbol{\theta})=\sum_{i=1}^{6}\left(\frac{\left(G_{n}^{\mathcal{D}}\left(F_{x, i}, \Omega \rightarrow 0\right)-G_{n}^{\mathcal{M}}\left(F_{x, i}, \Omega=0, \boldsymbol{\theta}\right)\right)^{2}}{\sigma_{\text {stat }}^{2}}\right) \\
& E_{\max , n}(\boldsymbol{\theta})=\sum_{i=1}^{6}\left(\frac{\left(G_{n}^{\mathcal{D}}\left(F_{x, i}, \Omega=\omega_{1}\right)-G_{n}^{\mathcal{M}}\left(F_{x, i}, \Omega=\omega_{1}, \boldsymbol{\theta}\right)\right)^{2}}{\sigma_{\max }^{2}}\right) \\
& E_{\mathrm{ef}, n}(\boldsymbol{\theta})=\sum_{i=1}^{6}\left(\frac{\left(\omega_{1, n}^{\mathcal{D}}\left(F_{x, i}\right)-\omega_{1, n}^{\mathcal{M}}\left(F_{x, i}, \boldsymbol{\theta}\right)\right)^{2}}{\sigma_{\mathrm{ef}}^{2}}\right)
\end{aligned}
$$

that are chosen to be the squared errors between experimental data and model predictions, cf. [25], for the static deflection $E_{\text {stat }}$, the maximum amplitude at resonance $E_{\max }$ and the first eigenfrequency $E_{\text {ef }}$ summed up over the six measured axial loads $F_{x, i}$. The variances $\sigma_{\text {stat }}^{2}, \sigma_{\max }^{2}$ and $\sigma_{\text {ef }}^{2}$ are related to the measurement errors of the corresponding measured values, that are assumed to be independent and normally distributed with zero mean [25]. In order to achieve reasonable results, the variances $\sigma_{\text {stat }}^{2}, \sigma_{\max }^{2}$ and $\sigma_{\text {ef }}^{2}$, which have a strong effect 
on the parameter calibration results, were chosen to be double the measurement errors of the corresponding measured values.

In this paper, the parameter space is explored using Markov Chain Monte CARLO (MCMC) sampling to approximate the posterior parameter distributions $P(\boldsymbol{\theta}, \mathcal{M} \mid \mathcal{D})$ by drawing multiple samples from these posterior parameter distributions. That is, the histograms of the parameters $\boldsymbol{\theta}$ of all random samples produce the approximated posterior parameter distributions $P(\boldsymbol{\theta}, \mathcal{M} \mid \mathcal{D})[25,28]$. By application of MCMC sampling, it is possible to circumvent the calculation of the denominator $P(\mathcal{D})$ in $(9)$ and, thus, to enable a mathematically more efficient application of the BAYESIAN inference parameter calibration approach using the Metropolis-Algorithm [25], as summarized in Algorithm 1.

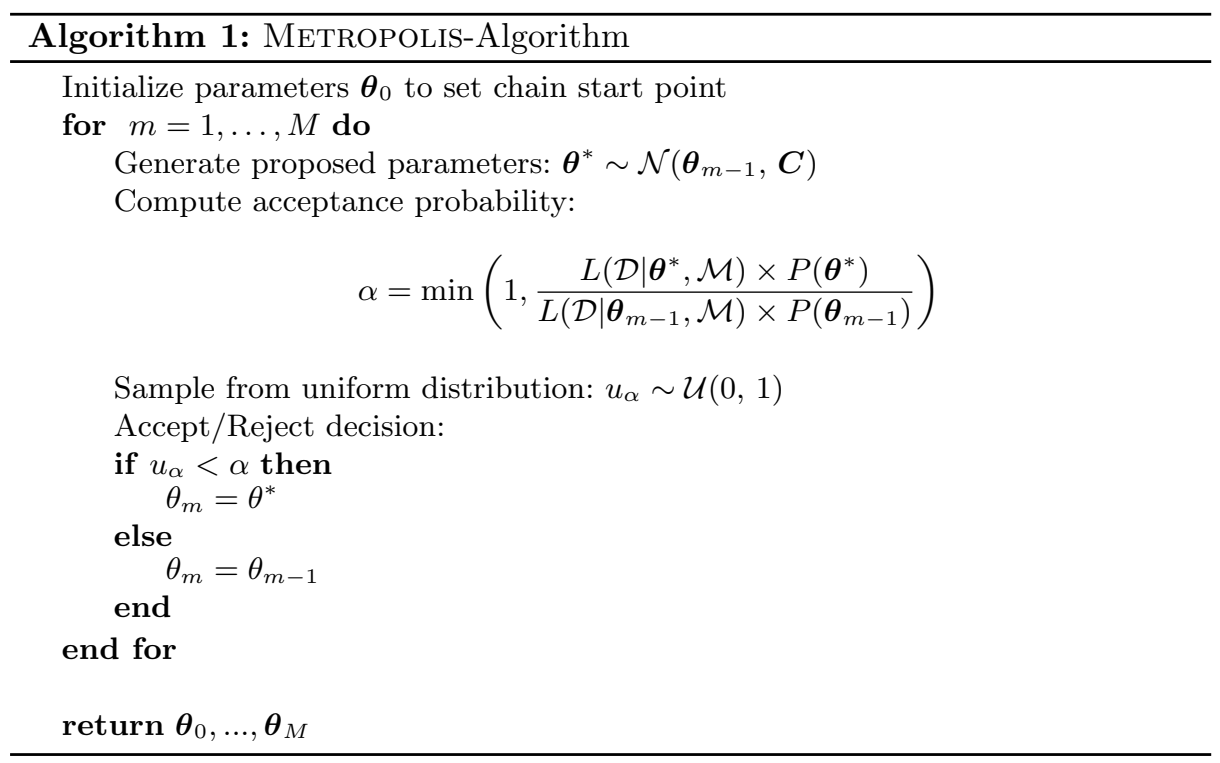

The number of $M$ chain elements is generated iteratively. The proposal distribution $\mathcal{N}\left(\boldsymbol{\theta}_{m-1}, \boldsymbol{C}\right)$ is the probability of moving to a point in the parameter space and is chosen to be GAUSSIAN centered at the current parameter set $\boldsymbol{\theta}_{m-1}$ with covariance matrix $\boldsymbol{C}$. The covariance matrix $\boldsymbol{C}$ governs the dispersion in terms of how far the proposal parameter set $\boldsymbol{\theta}^{*}$ moves from the current parameter set $\boldsymbol{\theta}_{m-1}$. Subsequently, the acceptance probability $\alpha$ is calculated by dividing the posterior probability given the proposed parameter set $\boldsymbol{\theta}^{*}$ by the posterior probability given the current parameter set $\boldsymbol{\theta}_{m-1}$. Each iteration ends with the decision, if the proposed parameter set $\boldsymbol{\theta}^{*}$ is accepted or the current parameter set $\boldsymbol{\theta}_{m-1}$ is kept. Each result is a sample of the posterior distributions and the histograms of the calibration candidate parameters for all samples represent the approximated posterior distributions. 
Figure 4 depicts the parameter calibration results obtained from $M=50.000$ MCMC runs, where an acceptance rate of $27 \%$ was achieved. On the diagonal, the parameter distributions are shown as histograms representing approximations of the marginal posterior distributions for the calibrated parameters $\boldsymbol{\theta}(7)$. The lower off-diagonal depicts distribution contour plots for the joint probability distribution pairs of the parameters. The upper off-diagonal depicts posterior sample pairs of the parameters. Furthermore, the narrow histograms graphically depict the knowledge gain and the uncertainty reduction of the posterior parameter ranges compared to the prior parameter bounds.

\subsection{Comparison of Calibration Results}

For a comparison and assessment of the uncertainty inherent to the calibration parameters, it is first advisable to examine the posterior distributions obtained by the forward parameter calibration and the BAYESIAN inference parameter calibration, as depicted in Fig. 3 and Fig. 4. In general, the posterior distributions obtained by the forward parameter calibration approach are more dense than those obtained by the BAYESIAN inference parameter calibration approach. This exception might have been provoked by the bad fitting of the normal distribution for $\zeta_{1}$, as can be seen in Fig. 3 .

Table 2 summarizes the prior and posterior uncertainty in form of parameter bounds and 95\% interpercentiles after parameter calibration as well as the reduction of parameter uncertainty in $\boldsymbol{\theta}(7)$. The parameter ranges covering the $95 \%$ interpercentiles are reduced by $42.3 \%$ to $79.0 \%$ for the forward parameter calibration approach and by $8.3 \%$ to $79.3 \%$ for the BAYESIAN inference parameter calibration approach compared to the prior bounds. Only for the modal damping ratio of the first mode of vibration $\zeta_{1}$, a larger reduction is achieved by the BAYESIAN inference approach.

While both approaches can be compared by the posterior distributions shown in Fig. 3 and Fig. 4, the posterior distribution obtained by BAYESIAN inference provides additional information about covariances displayed on the off-diagonals. These covariance plots can reveal statistical dependencies between the parameters. Inadequate modeling can cause these dependencies and help detect model uncertainty.

Aside from parameter uncertainty, the assessment and comparison of the model predictions for the axial load-dependent lateral dynamic behavior of the beam-column system provide valuable insights into the two calibration approaches. Figure 5 depicts the envelopes of amplitude $\left|G^{\mathcal{M}}\left(F_{x, i}, \Omega, \boldsymbol{\theta}\right)\right|$ and phase responses $\arg G^{\mathcal{M}}\left(F_{x, i}, \Omega, \boldsymbol{\theta}\right)$ for the prior distributions as well as both calibration approaches that were generated using 50.000 samples of the resulting calibrated parameters $\boldsymbol{\theta}$ within their $95 \%$ interpercentiles in Monte-Carlo simulations. Additionally, the envelopes for the 60 measured amplitude $\left|G^{\mathcal{D}}\left(F_{x, i}, \Omega\right)\right|$ and phase responses $\arg G^{\mathcal{D}}\left(F_{x, i}, \Omega\right)$ are shown. For the purpose of simplicity and clarity, the simulated and measured envelopes are only shown for the selected axial loads $F_{x, 1}=337 \mathrm{~N}, F_{x, 4}=1500 \mathrm{~N}$ and $F_{x, 6}=2500 \mathrm{~N}$. 


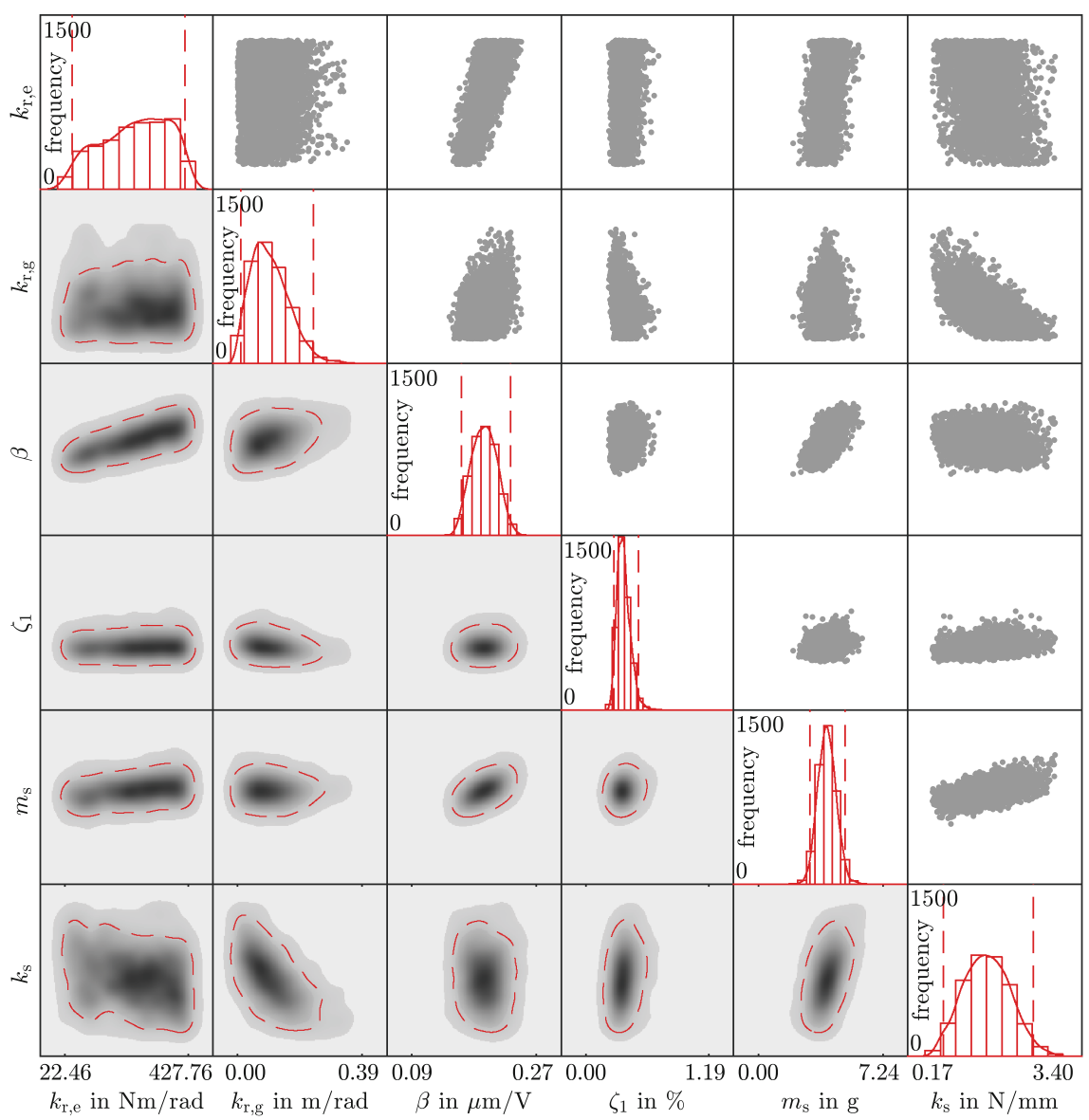

Fig. 4. Posterior distributions with $95 \%$ interpercentiles $(--)$ for the parameters $\boldsymbol{\theta}$ on the diagonal, bivariate joint distributions on the lower off-diagonal and posterior samples on the upper off-diagonal

Here, the area covered by the envelopes can be seen as a measure of prediction uncertainty. Compared to the envelopes generated using the prior bounds, the area covered by the posterior envelopes associated with the forward parameter calibration and the BAYESIAN inference parameter calibration approach is reduced by $43.0 \%$ and $86.7 \%$, respectively. The experimental data envelopes are almost entirely contained in the posterior envelopes and the axial loaddependency is well captured by both posterior envelopes. It is evident, that the BAYESIAN inference approach comes closest to the experimental data envelopes and, therefore, outperforms the forward parameter calibration approach. This assessment stands in contrast to the observations made for the parameter distributions that initially suggested a greater reduction of uncertainty for most 
Table 2. Prior and posterior uncertainty of the calibration parameters $\boldsymbol{\theta}(7)$ in form of bounds and $95 \%$ interpercentiles for the forward parameter calibration approach and the BAYESIAN inference approach as well as uncertainty reduction from prior bounds to $95 \%$ interpercentiles

\begin{tabular}{|c|c|c|c|c|c|c|c|c|}
\hline \multirow[t]{2}{*}{ Parameter } & \multicolumn{2}{|c|}{ Prior bounds } & \multicolumn{3}{|c|}{$\begin{array}{l}\text { Forward calibration } \\
95 \% \text { interpercentiles }\end{array}$} & \multicolumn{3}{|c|}{$\begin{array}{l}\text { BAYESIAN inference } \\
95 \% \text { interpercentiles }\end{array}$} \\
\hline & $\min$ & $\max$ & $\min$ & $\max$ & red & $\min$ & $\max$ & red \\
\hline$k_{\mathrm{r}, \mathrm{e}}$ in $\mathrm{Nm} / \mathrm{rad}$ & 22.46 & 426.76 & 113.81 & 347.23 & $42.3 \%$ & 45.84 & 416.40 & $8.3 \%$ \\
\hline$k_{\mathrm{r}, \mathrm{g}}$ in $\mathrm{m} / \mathrm{rad}$ & 0.00 & 0.39 & 0.01 & 0.14 & $68.3 \%$ & 0.01 & 0.24 & $42.5 \%$ \\
\hline$\beta$ in $\mu \mathrm{m} / \mathrm{V}$ & 0.09 & 0.27 & 0.15 & 0.21 & $67.8 \%$ & 0.16 & 0.23 & $62.0 \%$ \\
\hline$\zeta_{1}$ in $\%$ & 0.00 & 1.19 & 0.09 & 0.76 & $43.9 \%$ & 0.27 & 0.51 & $79.3 \%$ \\
\hline$m_{\mathrm{s}}$ in $\mathrm{g}$ & 0.00 & 7.24 & 2.99 & 4.51 & $79.0 \%$ & 3.01 & 5.03 & $72.1 \%$ \\
\hline$k_{\mathrm{s}}$ in $\mathrm{N} / \mathrm{mm}$ & 0.17 & 3.40 & 1.31 & 2.19 & $72.8 \%$ & 0.45 & 2.78 & $27.8 \%$ \\
\hline
\end{tabular}
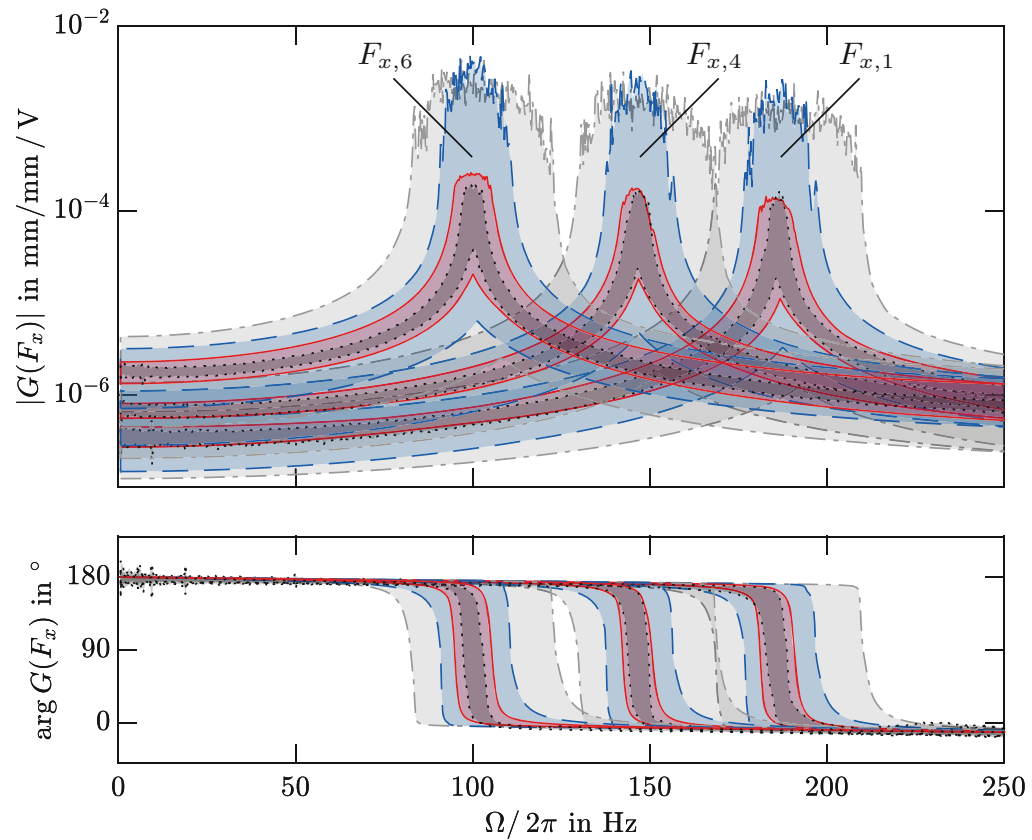

Fig. 5. Envelopes of amplitude and phase response obtained by sampling from prior distribution (ibration approach ([- $(\square)$ and the BAYESIAN inference approach ( $\square$ ) as well as envelope of the 60 measured amplitude and phase responses

parameters caused by the forward parameter calibration approach. This illustrates, that a reduction of parameter uncertainty alone is not able to indicate a reduction of prediction uncertainty. 


\section{Conclusion}

This paper presents two non-deterministic parameter calibration approaches for the model of a beam-column system to predict the lateral dynamic behavior. The beam-column system is intended to investigate active buckling control via piezoelastic supports. The parameters are calibrated to achieve model predictions that are statistically consistent with the experimental data. Therefore, instead of having a potentially over-fitted deterministic value for each calibrated parameter, the inherent uncertainty of the beam-column system is considered. The parameters to be calibrated are selected based on their uncertainty and sensitivity on the beam-column model predictions. Parameters with negligible uncertainty or sensitivity are not calibrated to reduce the computational effort and avoid identifiability issues. With the reduced number of parameters to be calibrated, the forward parameter calibration and the BAYESIAN inference parameter calibration are performed as non-deterministic parameter calibration approaches. The parameter ranges covering the $95 \%$ interpercentiles, indicating the parameter uncertainty, are reduced by $42.3 \%$ to $79.0 \%$ for the forward parameter calibration approach and by $8.3 \%$ to $79.3 \%$ for the BAYESIAN inference parameter calibration approach compared to the non-calibrated prior bounds. The parameter uncertainty of one parameter, the modal damping ratio of the first mode of vibration $\zeta_{1}$, is reduced significantly more by the BAYESIAN inference parameter calibration approach. This leads not only to a reduction of the uncertainty in the beam-column model predictions by up to $86.7 \%$ comparing to predictions obtained with non-calibrated prior bounds, but also to an almost identical representation of the experimental data. The forward parameter calibration approach leads to a reduction of the uncertainty in the beam-column model predictions by up to $43.0 \%$ compared to the prediction obtained with non-calibrated prior bounds with significantly wider envelopes around the experimental data. The BAYESIAN inference approach comes closest to the experimental data envelopes and, therefore, outperforms the forward parameter calibration approach. Nevertheless, by reducing the parameter uncertainty, the model prediction accuracy and credibility can be increased with both non-deterministic parameter calibration approaches. The consideration of model uncertainty in future investigations may further increase the accuracy and credibility of the beam-column model predictions. By that, biased results for the parameter calibration may be avoided by simultaneously keeping the physical meaning of the parameters.

Acknowledgement. The authors like to thank the Deutsche Forschungsgemeinschaft (DFG, German Research Foundation) for funding this project within the Sonderforschungsbereich (SFB, Collaborative Research Center) 805 "Control of Uncertainties in Load-Carrying Structures in Mechanical Engineering" - project number: 57157498. In addition, the authors acknowledge Lei Xu for his assistance during this project. 


\section{References}

1. Herold, S., Jungblut, T., Kurch, M.: A systematic approach to simulate active mechanical structures. In: Multi-Disciplinary Simulations - The Future of Virtual Product Development (2009)

2. Tamm, C., Thiel, J., Bartel, T., Atzrodt, H., Herold, S.: Methodisches vorgehen zur auslegung des vibro-akustischen verhaltens eines fahrzeugs. In: Wiedemann, M., Misol, M., Melz, T. (eds.) Smarte Strukturen und Systeme, pp. 95-106 (2016)

3. Platz, R., Götz, B.: Non-probabilistic uncertainty evaluation in the concept phase for airplane landing gear design. In: Barthorpe R.J., Platz, R., Lopez, I., Moaveni, B., Papadimitriou, C. (eds.) Model Validation and Uncertainty Quantification, Conference Proceedings of the Society for Experimental Mechanics Series, vol. 3. Springer International Publishing, Cham (2017)

4. Pelz, F.P., Pfetsch, M.E., Kersting, S., Kohler, M., Matei, A., Melz, T., Platz, R., Schaeffner, M., Ulbrich, S.: Types of Uncertainty, pp. 27-45. Springer International Publishing, Cham (2021). (in Print)

5. Green, P.L., Worden, K.: Modelling friction in a nonlinear dynamic system via Bayesian inference. In: Allemang, R., de Clerck, J., Niezrecki, C., Wicks, A. (eds.) Special Topics in Structural Dynamics, vol. 6, pp. 543-553. Springer, New York (2013)

6. Liu, D.-P.: Parameter identification for lugre friction model using genetic algorithms. In: Proceedings of 2006 International Conference on Machine Learning and Cybernetics, Piscataway NJ. IEEE (2006)

7. Mollineaux, M.G., van Buren, K.L., Hemez, F.M., Atamturktur, S.: Simulating the dynamics of wind turbine blades: Part i, model development and verification. Wind Energy 16(5), 694-710 (2013)

8. Wang, X., Lin, S., Wang, S.: Dynamic friction parameter identification method with lugre model for direct-drive rotary torque motor. In: Mathematical Problems in Engineering, pp. 1-8 (2016)

9. Wenjing, Z.: Parameter identification of lugre friction model in servo system based on improved particle swarm optimization algorithm, pp. 135-139 (2007)

10. Kennedy, M.C., O'Hagan, A.: Bayesian calibration of computer models. J. R. Stat. Soc. Ser. B (Stat. Methodol.) 63(3), 425-464 (2001)

11. Gehb, C.M.: Uncertainty evaluation of semi-active load redistribution in a mechanical load-bearing structure. Dissertation, Technische Universität Darmstadt, Darmstadt (2019)

12. Atamturktur, S., Hemez, F.M., Laman, J.A.: Uncertainty quantification in model verification and validation as applied to large scale historic masonry monuments. Eng. Struct. 43, 221-234 (2012)

13. Gehb, C., Atamturktur, S., Platz, R., et al.: Bayesian Inference Based Parameter Calibration of the LuGre-Friction Model. Exp Tech 44, 369-382 (2020). https:// doi.org/10.1007/s40799-019-00355-7

14. Schaeffner, M.: Quantification and evaluation of uncertainty in active buckling control of a beam-column subject to dynamic axial loads. Dissertation, Technische Universität Darmstadt, Darmstadt (2019)

15. Schaeffner, M., Platz, R.: Gain-scheduled $H_{\infty}$ buckling control of a circular beamcolumn subject to time-varying axial loads. Smart Mater. Struct. 27(6), 065009 (2018)

16. van Buren, K.L., Mollineaux, M.G., Hemez, F.M., Atamturktur, S.: Simulating the dynamics of wind turbine blades: Part ii, model validation and uncertainty quantification. Wind Energy 16(5), 741-758 (2013) 
17. Enss, G.C., et al.: Device for bearing design elements in lightweight structures (Festkörperlager). DE 102015101084 A1

18. Fuller, C.R., Elliott, S.J., Nelson, P.A.: Active Control of Vibration. Academic Press Inc. (1997)

19. Ljung, L.: System Identification Toolbox ${ }^{T M}$. User's Guide. Natick, MA, MathWorks (2017)

20. Preumont, A.: Vibration control of active structures, An introduction. Springer, Berlin (2011)

21. Skogestad, S., Postlethwaite, I.: Multivariable Feedback Control. John Wiley \& Sons (2001)

22. Schaeffner, M., Platz, R., Melz, T.: Adequate mathematical beam-column model for active buckling control in a tetrahedron truss structure. In: Model Validation and Uncertainty Quantification, Conference proceedings of the Society for Experimental Mechanics series, vol. 3, pp. 323-332. Springer International Publishing (2020)

23. Li, S., Goetz, B., Schaeffner, M., Platz, R.: Approach to prove the efficiency of the Monte Carlo method combined with the elementary effect method to quantify uncertainty of a beam structure with piezo-elastic supports. In: Papadrakakis, M., Papadopoulos, V., Stefanou, G. (eds.) Proceedings of the 2nd International Conference on Uncertainty Quantification in Computational Sciences and Engineering (UNCECOMP 2017), pp. 441-455. Institute of Structural Analysis and Antiseismic Research School of Civil Engineering National Technical University of Athens (NTUA) (2017)

24. Mathworks Inc., ed. Optimization toolbox ${ }^{T M}$, User's Guide. Natick, MA, MathWorks (2018)

25. Smith, R.C.: Uncertainty quantification: theory, implementation, and applications. Computational Science Engineering. Society for Industrial and Applied Mathematics, Philadelphia (2014). ISBN 978-1-611973-21-1

26. Nagel, J.B.: Bayesian techniques for inverse uncertainty quantification. Dissertation, ETH Zürich (2017)

27. Bayes, T.: An essay towards solving a problem in the doctrine of chances. By the late Rev. Mr. Bayes, F.R.S communicated by Mr. Price, in a letter to John Canton, A.M.F.R.S Philosophical Transactions of the Royal Society of London, vol. 53, pp. 370-418 (1763)

28. Riddle, M., Muehleisen, R.T.: A guide to Bayesian calibration of building energy models. In: ASHRAE/IBPSA-USA (2014) 
Open Access This chapter is licensed under the terms of the Creative Commons Attribution 4.0 International License (http://creativecommons.org/licenses/by/4.0/), which permits use, sharing, adaptation, distribution and reproduction in any medium or format, as long as you give appropriate credit to the original author(s) and the source, provide a link to the Creative Commons license and indicate if changes were made.

The images or other third party material in this chapter are included in the chapter's Creative Commons license, unless indicated otherwise in a credit line to the material. If material is not included in the chapter's Creative Commons license and your intended use is not permitted by statutory regulation or exceeds the permitted use, you will need to obtain permission directly from the copyright holder. 